MEDICAL MEMORANDA

\section{Severe Yersinia Pseudotuberculosis Infection Diagnosed at Laparoscopy}

\author{
M. J. BRODIE, P. A. BOOT, R. W. A. GIRDWOOD
}

British Medical fournal, 1973, 4, 88

There has been increased awareness in recent years of the association between chronic hepatic cirrhosis and severe infection with Yersinia (Pasteurella) pseudotuberculosis. Marlon et al. (1971) described two cases of $Y$. pseudotuberculosis septicaemia and reviewed 21 previously documented cases. Of 10 cases with chronic hepatic cirrhosis and haemochromatosis only one survived. Yamashiro et al. (1971) reported a further survival in a patient with haemochromatosis. We report survival in a case of severe $Y$. pseudotuberculosis infection associated with haemochromatosis, where diagnosis was made by laparoscopy.

\section{Case Report}

A 38-year-old ships' surveyor was admitted to hospital in severe diabetic ketoacidosis. He gave a history of polyuria and polydipsia of one year's duration which had worsened in the previous two weeks. There was no history of abdominal pain or upset in bowel habit. He was noted to be pyrexial on admission but bacteriological investigations including seven blood cultures over 10 days were negative. The diabetic ketosis responded to the administration of soluble insulin and parenteral fluids and he was rapidly stabilized on a single injection of insulin zinc suspension (Lente).

Four days after admission he remained markedly pyrexial and hepatomegaly became apparent. Isotopic liver scanning suggested a diffuse abnormality. Nine days after admission biochemical evidence of hepatocellular failure became evident and liver biopsy was performed. Sections showed a macronodular cirrhosis with severe siderosis consistent with haemochromatosis. Serum iron at that time was normal. Over the next few days his condition gradually deteriorated and he became anaemic and cachectic. Hepatocellular failure progressed and he developed ascites. Three weeks after admission, because of further unexplained deterioration, laparoscopy was performed. This showed a cirrhotic liver with several small abscesses on the upper surface of the right lobe. A biopsy specimen of one of these was taken and a swab of the pus was obtained. Therapy with large doses of intravenous ampicillin $(4 \mathrm{~g} /$ day $)$ and cloxacillin $(8 \mathrm{~g} /$ day $)$ was begun. $Y$. pseudotuberculosis was isolated from the abscess swab.

The patient responded rapidly to antibiotic therapy, becoming apyrexial within 48 hours. Hepatic function progressively improved with subsidence of ascites and reduction in hepatomegaly. Liver function tests also showed gradual improvement. Oral antibiotics were substituted for parenteral after a further three weeks, and he was well enough to return home six weeks after admission. He was well three months after discharge, though routine liver function tests remained slightly abnormal. The serum iron and percentage saturation rose consistent with a diagnosis of haemochromatosis, which was confirmed by a desferrioxamine test.

BACTERIOLOGY

Cultures from the liver abscess fluid produced a growth of Gramnegative pleomorphic bacilli. The organism had the morphological,

Stobhill General Hospital, Glasgow G21 3UW

M. J. BRODIE, M.B., CH.B., Senior House Officer in Medicine

P. A. BOOT, F.I.M.L.T., Senior Chief Technician

R. W. A. GIRDWOOD, M.R.C.PATH., Locum Consultant Bacteriologist cultural, and biochemical characteristics of $Y$. pseudotuberculosis with the exception that it did not ferment arabinose and salicin even after prolonged incubation. No motility occurred at $37^{\circ} \mathrm{C}$ but was readily found at $18-22^{\circ} \mathrm{C}$. Two guinea-pigs were inoculated intraperitoneally with $0.5 \mathrm{ml}$ of a 24 -hour broth culture of the organism. One animal died after 48 hours and the other after 96 hours. At necropsy the latter showed a small macroscopic nodule in the liver. The organism was recovered from the liver, lungs, and spleen of both animals and histological examination showed multiple abscesses in all these organs. Numerous Gram-negative bacilli were present surrounded by acute inflammatory cells.

The patient's serum was tested against freshly prepared antigens from the organism and agglutination occurred at a titre of $1 / 132$ three weeks after admission to hospital. When tested three weeks later the titre had fallen to $1 / 132$. An indirect fluorescent test was also applied to these sera and was strongly positive on the first sample but only weakly positive on the second. Agglutination tests performed on his wife and daughter were negative. The organism was sensitive to ampicillin, cotrimoxazole, tetracycline, kanamycin, cephalexin, cephaloridine, and partially sensitive to penicillin (minimal inhibitory concentration 0.75 units $/ \mathrm{ml}$ ). The organism was sent to Dr. N. S. Mair, Public Health Laboratory, Groby Road Hospital, Leicester, who confirmed it to be $Y$. pseudotuberculosis subtype Ib.

\section{Comment}

Y. (Pasteurella) pseudotuberculosis is a common animal pathogen. It causes pseudotuberculosis in guinea-pigs, field mice, rats, rabbits, and other mammals, and it has also been isolated from birds. In man the most frequent presentation of $Y$. pseudotuberculosis is acute mesenteric lymphadenitis, but it occasionally presents as an overwhelming generalized infection. The portal of entry in both forms is presumed to be the bowel, and Conn (1964) postulates that in the cirrhotic patient the organism may bypass the reticuloendothelial system of the damaged liver resulting in a bacteraemia and metastatic abscess formation. Despite lack of bowel symptomatology it seems likely that in the present case the organism reached the liver via the portal system, as blood cultures were persistently negative and there was no evidence of metastatic abscess formation in any other organ. The source of infection would seem to be from infected rodent excreta encountered in his profession as a ships' surveyor.

A definitive diagnosis would almost certainly not have been made in this case in the absence of exploratory laparoscopy. The use of the laparoscope in severe liver disease of obscure origin offers a simple and relatively safe method of investigation and can be performed with the minimum of risk in the presence of ascites. McAuley et al. (1967) emphasized the importance of examining patients with obscure septicaemic disease for Pasteurella antibodies. We would like to stress the possibility of $Y$. pseudotuberculosis infection in any patient with known chronic hepatic cirrhosis who presents with a severe infective illness.

We would like to thank Dr. M. G. Dunnigan for permission to report this case. We gratefully acknowledge the help of Mr. F. T. Crossling, who performed the laparoscopy, of Messrs. I. Howie and L. Smart for technical help, and of Dr. N. S. Mair in confirming the identity of the organism.

\section{References}

Conn, H. O. (1964). Annals of Internal Medicine, 60, 568. McAuley, J. D., Wilson, J. A. C., Abbott, J. D., and Mair, N. S. (1967). British Medical fournal, 2, 553.

Mair, N. S. (1968). Recent Advances in Clinical Pathology: Series V. Churchill.

Marlon, A., Gentry, L., and Merigan, T. C. (1971). Archives of Internal Medicine, 127, 947 .

Yamashiro, K. M., Goldman, R. H., Harris, D., and Uyeda, C. T. (1971). Archives of Internal Medicine, 128, 605 . 